
\title{
28 Research Square \\ Trends in Hysteroscopic Endometrial Sampling in Women 70 Years and Older
}

\section{Shikma Bar-On}

Tel Aviv Sourasky Medical Center

\section{Neta Solomon}

Tel Aviv Sourasky Medical Center

\section{Gilad Rattan}

Tel Aviv Sourasky Medical Center

\author{
Alon Ben- David \\ Sheba Medical Center \\ Dan Grisaru ( $\sim$ grisaro@tauex.tau.ac.il ) \\ Tel Aviv Sourasky Medical Center
}

\section{Research Article}

Keywords: endometrial cancer, endometrial hyperplasia, general anesthesia, menopause, office hysteroscopy

Posted Date: August 4th, 2021

DOl: https://doi.org/10.21203/rs.3.rs-661371/v1

License: (c) (i) This work is licensed under a Creative Commons Attribution 4.0 International License. Read Full License 


\section{Abstract}

Background: Women 70 years of age or older with suspected endometrial pathologies would especially benefit from office hysteroscopy due to the risks of anesthesia and high rates of clinically significant findings in this age group. However, performing office hysteroscopy in those older patients can be technically challenging, and patient cooperation, which is essential for the success of the office procedure, may be compromised in this age group. The objective of the present study was to characterize the clinical and procedural features of office and operating-room hysteroscopy in women 70 years of age or older.

Methods: This was a retrospective chart analysis of all women aged 70 years and older who underwent a hysteroscopy procedure in a single tertiary-care medical center between March 2011 and August 2018. We compared the clinical and procedural characteristics of patients who underwent hysteroscopy in our outpatient clinic to those who underwent hysteroscopy under anesthesia in the operating room. Primary outcomes included procedure success and histopathological findings. Secondary outcomes included short-term complications of the procedure.

Results: The data of 577 patients were analyzed. The median age at the time of the procedure was 76 years. 225 office hysteroscopy procedures and 405 operating room procedures were included. Of the 236 patients with postmenopausal bleeding (PMB), 73 (30.9\%) were diagnosed with having malignancy compared to $25 / 341(7.3 \%)$ patients with no PMB $(p<.001$, odds ratio $(O R)=5.66$; confidence interval (CI) 3.46-9.26). The office procedure was successful in 194 (87\%) patients. Fifty-three patients required a second hysteroscopy, which revealed 12 cases of premalignant or malignant lesions. The volume of procedures in the office increased 16 times during the study period, and the diagnostic yield of new abnormal findings by means of the procedure in the office setting has increased over time. Operating room hysteroscopy had a higher rate of complications compared to office hysteroscopy, including mortalities related to the procedure.

Conclusions: Office hysteroscopy for women over the age of 70 is safe, feasible, and reliable for diagnosis of endometrial malignancy. Failed hysteroscopy and a moderate-to-high index of suspicion for malignancy indicates the need for a repeat hysteroscopy.

\section{Introduction}

Abnormal uterine bleeding, suspected endometrial polyps, and endometrial thickness are sources of concern among postmenopausal women that require tissue biopsy of the endometrial finding $[1,2]$. Hysteroscopy is the gold standard for diagnosis [3,4] and treatment [5] of intrauterine abnormality. Traditionally, hysteroscopy is carried out under general anaesthesia. However, in recent years, major technological improvements, such as the availability of high-resolution, small-diameter endoscopes [6], the use of saline as distension medium [7] and the development of an atraumatic insertion technique [8] have increased the feasibility and acceptability of outpatient diagnostic and therapeutic hysteroscopy. 
These developments have made it possible to combine diagnostic and operative hysteroscopy in an outpatient setting without anesthesia (the "see-and-treat" technique).

The "see-and-treat" technique, which offers numerous advantages and has gained increasing popularity in recent years, is particularly useful for postmenopausal women, due to the higher rates of comorbidities and higher risks associated with anesthesia in this age group. The "see-and-treat" technique is also often preferred by these older patients and their families, with higher probability of these patients deciding not to undergo general anesthesia when given the choice to avoid it.

However, in this group of postmenopausal women, a number of factors may discourage a trial of office procedure. These factors include the technical challenge of cervical stenosis, bleeding due to anticoagulation treatment or the absence of an anesthesiologist in cases of unpredicted complications. Moreover, musculoskeletal limitations such as osteoarthritis of the hips, hearing impairments or mental disorders that cause cognitive impairment can compromise patient positioning and cooperation, which are essential for the success of the "see and treat" procedure.

We previously evaluated the accuracy of outpatient hysteroscopy among peri- and postmenopausal women and showed it to be an adequate and reliable tool mainly for the evaluation of benign pathology, with a sensitivity of $93.1 \%$, a specificity of $52.1 \%$, a positive predictive value of $90.4 \%$, and a negative predictive value of $61 \%$ for a visual diagnosis of any pathology [9].

Most studies dealing with office hysteroscopy and menopause examined the group of post-menopausal women as a whole $[4,10]$. However, the present study focused on women 70 years and older, given the combination of higher risks of anesthesia and the higher rates of clinically significant findings in that group. The aim of this study is to characterize both office hysteroscopy and operating room (OR) hysteroscopy for the diagnosis of endometrial pathologies. In addition, we aimed to illustrate trends in the utility of hysteroscopy techniques and in the detection of abnormal findings in this age group over a period of 7 years.

\section{Material And Methods}

\section{Study Population}

This study was approved by the Research Ethics Committee at the Tel Aviv Sourasky Medical Center (0749-15-TLV on 10.11.2016). We used the data on all consecutive women 70 years of age or older who were referred to our medical center for a hysteroscopy procedure between March 2011 and August 2018. Data for patients in the office hysteroscopy group were retrieved from the hysteroscopy clinic's medical charts, and patients in the OR hysteroscopy group were identified according to their OR diagnosis (ICD codes 68.12 and 68.16). All hysteroscopic interventions that had been performed during the study period were reviewed, including repeat hysteroscopies for the same patient. Cases of repeat hysteroscopic procedures for the same patient that were not related to her primary procedure were excluded from the 
analysis. All the patients referred to either office or OR hysteroscopy had undergone at least one gynecological examination that included an ultrasound study before their referral to our unit.

Women were assigned to the postmenopausal bleeding (PMB) group if they presented with PMB, regardless of ultrasonographic findings. Women who were referred due to any ultrasound finding with no PMB were assigned to the abnormal sonographic findings group.

The "see-and-treat" technique was implemented in our institution with the opening of a new outpatient hysteroscopy clinic in 2011. As a result, a change of clinical practice of referring women to the office instead of directly to the OR as a first-line procedure was gradually introduced in our medical community.

\section{Procedures}

All methods were performed in accordance with the relevant guidelines and regulations. Office procedures were performed without anesthesia. The surgeon used narrow caliber hysteroscopes, i.e., a $3.8 \mathrm{~mm}$ telescope with a 30-degree oblique lens and a 5-Fr working channel (compact hysteroscope; Richard Wolf $\mathrm{GmbH}$, Knittlingen Germany), a $1.9 \mathrm{~mm}$ scope with a $3 \mathrm{~mm}$ diagnostic sheath (Mini-Hysteroscopes, Olympus Corporation, Hamburg, Germany) and a $1.8 \mathrm{~mm}$ telescope with $3.5 \mathrm{~mm}$ diagnostic sheath with a 5F operative canal (Alphascope; Gynecare, division of Ethicon, Inc., Somerville, NJ). Illumination was provided by a high-intensity cold-light source via a fiberoptic lead. The uterine cavity was distended with normal saline infused by a pressure bag at a pressure of $50-100 \mathrm{mmHg}$. The vaginoscopic approach was used in all cases. Polypectomy was performed with a bipolar coaxial electrode (Gynecare VersaPoint, Ethicon Inc., Somerville, NJ). Endometrial biopsies were performed by means of a bipolar electrode, flexible scissors, or grasping forceps. All procedures were performed by two gynecologists (SB and GR).

OR procedures were performed under anesthesia and by means of either a $3.8 \mathrm{~mm}$ telescope with a 30degree oblique lens and a 5Fr working channel (compact hysteroscope; Richard Wolf GmbH, Knittlingen, Germany) or a Versapoint 2 bipolar resectoscope 24Fr (J\&J, Germany). The uterine cavity was distended with a Stortz Endomat Select hysteroscopy fluid-management system set to a pressure of approximately $100 \mathrm{mmHg}$.

The hysteroscopy was defined as successful only if penetration through the cervical internal os was achieved and the entire uterine cavity was visualized. The hysteroscopy was defined as failure when no penetration of the uterine cavity was achieved. It was defined as incomplete when the surgeon could not complete the procedure in one step, e.g., partial resection of an endometrial polyp for which additional office or OR procedures were usually recommended. Incomplete procedures were counted among the successful procedures.

Data were collected by reviewing the computerized medical records with a de-identified pre-prepared data sheet. All of the study patients' computerized records were searched for follow-up information until the end of the study period.

\section{Statistical Analysis}


Nominal data were described as numbers and percentages. Continuous data were assessed for normal distribution (Shapiro-Wilk test) and provided as the mean \pm standard deviation or median $\left(P_{25}-P_{75}\right)$. Quantitative data were analyzed with the chi-square or Fisher exact test. Continuous variables were compared between groups by means of the t-test or the Mann-Whitney nonparametric test, as appropriate. All statistical tests were two sided, with $p<0.05$ considered statistically significant. Data analyses were conducted with the Statistical Package for the Social Sciences, version 25.0 (IBM SPSS Statistics for Windows, Version 25.0. Armonk, NY: IBM Corp) and Microsoft Excel version 14.0 (Microsoft Corporation, Redmond, Washington).

\section{Results}

A total of 642 hysteroscopies for 577 patients aged 70 years and older were performed during the study period. The median patient age at the time of the procedure was 76 years (interquartile range [IQR] 7380). Two-hundred and twenty-two of the patients were included in the office-hysteroscopy group, and 355 patients were included in the OR hysteroscopy group.

Two-hundred and thirty hysteroscopies were performed in the outpatient hysteroscopy clinic, and 412 hysteroscopies were performed in the OR. Twelve patients underwent repeat hysteroscopic procedures unrelated to the primary procedure. In these cases, only the second procedure was included in the analysis. The final analysis consisted of a total of 630 hysteroscopies, of which 225 were performed in the office and 405 in the OR. Five-hundred seventy-seven hysteroscopies were first interventions and another 53 were second interventions.

Figure 1A illustrates the volume of procedures among the two groups of women during the study period. While the total number of hysteroscopies more than doubled during this period, the number of procedures for this population in the outpatient hysteroscopy clinic increased by approximately 16 times. Thus, by the end of the follow-up period, an office procedure became the primary hysteroscopic intervention for patients 70 years of age or older.

The study population included 416 (72.1\%) patients aged 70-79 years, $155(26.9 \%)$ patients aged 80-89 years, and six (1.0\%) patients aged 90 years and over (the oldest was 93 years old). The baseline characteristics of the two groups are detailed in Table 1. The median age at the time of hysteroscopy (similar for the two groups) was 76 years (IQR 73-80). More women were referred to office procedures due to abnormal sonographic findings $(67.1 \%$ vs. $49.6 \%, p<0.001$ : Table 1$)$, while more women with active PMB were referred to procedures in the OR $(47 \%$ vs. $31.1 \% \mathrm{p}<0.001$ : Table 1$)$. 
Table 1

Preoperative and operative characteristics

\begin{tabular}{|c|c|c|c|}
\hline \multirow[t]{3}{*}{ Variable } & \multicolumn{2}{|c|}{ Type of hysteroscopy } & \multirow[t]{3}{*}{$\mathrm{p}$-value } \\
\hline & Office & OR & \\
\hline & $(n=222)$ & $(n=355)$ & \\
\hline Age, years, median (IQR) & $76(70-91)$ & $76(71-93)$ & 0.241 \\
\hline \multicolumn{4}{|l|}{ Indications for hysteroscopy } \\
\hline Post-menopausal bleeding, n (\%) & $69(31.1)$ & $167(47.0)$ & $<0.001$ \\
\hline Abnormal sonographic findings ${ }^{a}, \mathrm{n}(\%)$ & $149(67.1)$ & $176(49.6)$ & $<0.001$ \\
\hline Other indications ${ }^{\mathrm{b}}, \mathrm{n}(\%)$ & $4(1.8)$ & $12(3.4)$ & 0.308 \\
\hline Successful procedure, n (\%) & $194(87.4)$ & $342(96.3)$ & $<0.001$ \\
\hline Incomplete procedure, n (\%) & $45(20.3)$ & $6(1.7)$ & $<0.001$ \\
\hline \multicolumn{4}{|l|}{ Main findings during procedure } \\
\hline Endometrial polyp, n (\%) & $122(55.0)$ & $191(53.8)$ & 0.797 \\
\hline Thickened endometrium, $\mathrm{n}(\%)$ & $16(7.2)$ & $70(19.7)$ & $<0.001$ \\
\hline Atrophic endometrium, n (\%) & $39(17.6)$ & $80(22.5)$ & 0.170 \\
\hline Submucosal leiomyoma, n (\%) & $19(8.6)$ & $27(7.6)$ & 0.752 \\
\hline Adhesions, n (\%) & $16(7.2)$ & $17(4.8)$ & 0.269 \\
\hline Vaginal or cervical polyps, n (\%) & $8(3.6)$ & $7(2)$ & 0.284 \\
\hline Cervical stenosis, n (\%) & $54(24.3)$ & $52(14.6)$ & 0.004 \\
\hline \multicolumn{4}{|l|}{ Main types of intervention } \\
\hline Total operative hysteroscopy, n (\%) & $134(60.4)$ & $335(94.4)$ & $<0.001$ \\
\hline Hysteroscopic polypectomy, n (\%) & $98(44.1)$ & $190(53.5)$ & 0.032 \\
\hline Hysteroscopic myomectomy, n (\%) & $13(5.8)$ & $28(7.9)$ & 0.355 \\
\hline Tissue sampling, n (\%) & $33(14.9)$ & $123(34.6)$ & $<0.001$ \\
\hline Removal of IUD, $\mathrm{n}(\%)$ & $2(0.9)$ & $4(1.1)$ & $>0.999$ \\
\hline
\end{tabular}




\begin{tabular}{|c|c|c|c|}
\hline \multirow[t]{3}{*}{ Variable } & \multicolumn{2}{|c|}{ Type of hysteroscopy } & \multirow[t]{3}{*}{ p-value } \\
\hline & Office & OR & \\
\hline & $(n=222)$ & $(n=355)$ & \\
\hline \multicolumn{4}{|c|}{ Main reasons for failure or incomplete procedure } \\
\hline Cervical stenosis, n (\%) & $24(10.8)$ & $11(3.1)$ & $<0.001$ \\
\hline Pain or intolerance, $\mathrm{n}(\%)$ & $7(3.15)$ & $0(0)$ & 0.001 \\
\hline Large/multiple findings or bleeding, $n(\%)$ & $35(15.8)$ & $2(0.6)$ & $<0.001$ \\
\hline Uterine perforation, $\mathrm{n}(\%)$ & $1(0.5)$ & $2(0.6)$ & 0.856 \\
\hline Other failure ${ }^{\mathrm{c}}, \mathrm{n}(\%)$ & $6(2.7)$ & $4(1.3)$ & 0.158 \\
\hline \multicolumn{4}{|c|}{$\begin{array}{l}\text { a Including } 5 \mathrm{~mm} \text { or more thickened endometrium, suspected endometrial polyp, submucosal } \\
\text { leiomyoma, or fluid in uterine cavity. }\end{array}$} \\
\hline \multicolumn{4}{|c|}{ b Retained IUD $(n=1)$, vaginal secretions $(n=1)$, metastatic workup $(n=10)$, vaginal lesions $(n=4)$. } \\
\hline \multicolumn{4}{|c|}{${ }^{c}$ Blocking cervical mass $(n=5)$, vaginal stenosis $(n=1)$, uterine prolapse $(n=1)$, improper } \\
\hline
\end{tabular}

The procedure was successful in 194 (87.4\%) patients who underwent the procedure in the office compared with 342 (96.3\%) patients who underwent the procedure in the OR under anesthesia. The diagnosis of cervical stenosis or difficulty in penetrating the cervix was significantly higher in the office group. Out of $54(24.3 \%)$ cases in the office group with cervical stenosis, the surgeon managed to enter the uterine cavity in 32 (59.2\%). The surgeon failed to penetrate the uterine cavity, mainly due to cervical stenosis, obstructive cervical mass, and patient intolerance, in $28(12.6 \%)$ of office hysteroscopies. Fortyfive $(20.3 \%)$ of the hysteroscopies in our clinic were not completed in a one-step procedure, mainly due to large or multiple findings and uterine bleeding. Only 7 (3.15\%) procedures were terminated due to patient discomfort.

A total of 47 (21.2\%) patients who underwent office hysteroscopy required a second hysteroscopy (19 due to failure in the first visit, 27 due to incomplete procedures, and one due to continuous bleeding that precluded the performance of the intervention). In 44 of those 47 cases, the second hysteroscopy was performed in the OR under general anesthesia. A total of six patients who underwent OR hysteroscopy under general anesthesia required a second procedure (two cases due to failure and four cases due to non-diagnostic histopathologic samples taken at the first procedure).

Overall, $150(67.6 \%)$ of office hysteroscopies had a positive finding. Moreover, $43(43 / 69=62.3 \%)$ of the women with PMB and $106(106 / 149=71.1 \%)$ of those with abnormal sonographic findings also had a 
positive hysteroscopic finding(s). The histopathological findings by groups are shown in Table 2. PMB was a risk factor for malignancy for the entire cohort, with 73/236 (30.9\%) patients with PMB diagnosed as having a malignancy, compared to $25 / 341(7.3 \%)$ patients with no PMB $(p<.001$, odds ratio $(O R)=$ 5.66; confidence interval (Cl) 3.46-9.26). In the repeat hysteroscopy subgroup, 7/17 (41.2\%) patients with PMB were diagnosed as having a malignancy compared to 6/36 (16.7\%) patients with no bleeding ( $p$ $=.053$, odds ratio $(\mathrm{OR})=3.5$; confidence interval $(\mathrm{Cl})$ 0.95-12.89).

Table 2

Histopathologic findings

\begin{tabular}{|c|c|c|c|c|}
\hline \multirow[t]{3}{*}{ Variable } & \multicolumn{3}{|c|}{ Type of hysteroscopy } & \multirow[t]{3}{*}{ p-value } \\
\hline & Office & OR & Repeat & \\
\hline & $(n=222)$ & $(n=355)$ & $(n=53)$ & \\
\hline Tissue sampling during procedure, $\mathrm{n}(\%)$ & $130(58.6)$ & $335(94.4)$ & $48(90.6)$ & $<0.001$ \\
\hline \multicolumn{5}{|l|}{ Histologic report, n (\%) } \\
\hline Benign & $107(82.3)$ & $228(68.1)$ & $35(72.9)$ & 0.009 \\
\hline Abnormal & $19(14.6)$ & $79(23.6)$ & $12(25.0)$ & 0.087 \\
\hline Premalignant $^{\mathrm{a}}$ & $6(4.6)$ & $16(4.8)$ & $3(6.2)$ & \\
\hline Malignant ${ }^{\mathrm{b}}$ & $13(10)$ & $63(18.8)$ & $9(18.8)$ & \\
\hline Non-diagnostic & $4(3.1)$ & $28(8.3)$ & $1(2.1)$ & 0.050 \\
\hline \multicolumn{5}{|c|}{$\begin{array}{l}\text { a Including hyperplasia or complex hyperplasia without atypia }(n=13) \text {, } \\
\text { metaplasia/hyperplasia/complex hyperplasia with atypia }(n=10) \text {, atypical leiomyoma }(n=1) \text {, } \\
\text { intrafallopian intraepithelial serous adenocarcinoma }(n=1) \text {. }\end{array}$} \\
\hline \multicolumn{5}{|c|}{$\begin{array}{l}\text { b Including endometrial carcinoma (Figo } 1-3 ; n=63) \text {, uterine sarcoma }(n=10) \text {, squamous cell } \\
\text { carcinoma of vagina or cervix }(n=8) \text {, metastases (primary melanoma, colorectal carcinoma; } n=4) \text {. }\end{array}$} \\
\hline
\end{tabular}

Figure 1B illustrates the relative contribution of each of the hysteroscopic approaches to the diagnosis of new abnormal findings among the 577 patients included in our cohort.

While samples taken in the outpatient hysteroscopy clinic during the first years did not contribute to a new diagnosis, their contribution increased during the following years.

All patients who underwent hysteroscopy in our outpatient clinic were discharged on the same day. The median length of hospitalization was three days (range 0-28 days) for the patients who underwent the procedures in the OR. Three-hundred and twenty-nine cases ( $92.7 \%$ of all procedures under anesthesia) were performed in an inpatient setting, while 26 (7.3\% of all procedures under anesthesia) were performed in our outpatient ORs. 
Short-term complications among patients treated in our outpatient hysteroscopy clinic included one case $(0.45 \%)$ of uterine perforation that was managed expectantly, and one case of bleeding that necessitated admission a few days later for a repeat procedure under general anesthesia. Four of the $355(1.13 \%)$ patients who underwent an OR hysteroscopy sustained uterine perforation. Emergent abdominal surgeries were required in three of those cases and included two abdominal hysterectomies, including one case of left colectomy. Three patients $(0.85 \%)$ sustained complications that required admission to the intensive care unit (ICU): one cardiac arrest, and two cases of severe sepsis (secondary to pyometra). Unfortunately, the latter two patients died during the hospitalization, yielding a mortality risk of $0.56 \%$ for patients aged 70 years and older in our cohort who underwent OR hysteroscopy.

\section{Discussion}

Referring a patient 70 years of age or older to either an outpatient hysteroscopy or to an OR procedure under general anesthesia is a clinical dilemma, given the combination of higher risks of anesthesia and the various factors which may discourage a trial of office procedure in that age group. The considerations which may affect this clinical decision were briefly summarized in the introduction above.

Altogether, 194 (87.4\%) patients in our cohort who were referred to office hysteroscopy had a successful procedure. Our results showed a success rate similar to the $74.5 \%$ success rate for office hysteroscopy in postmenopausal women recently reported by Sauvan et al. [11]. Sousa et al., who used a hysteroscopic technique similar to ours on 88 postmenopausal patients with genital bleeding, reported similar failure rates of $17.8 \%$ [12].

Endometrial sampling was generally less likely to be performed in the office compared to the OR, but the fact that more than one-half of the women in our cohort (58.6\%) did have a biopsy taken during the office procedure is of considerable diagnostic value for this age group. It is important to emphasize that even incomplete procedures, which were much more common in the office group than in the OR group, are of some value in this age group, especially if a biopsy has been carried out. For example, if a biopsy from a benign endometrial polyp is taken, the patient and her physician would feel more confident about choosing a conservative sonographic follow-up, thus avoiding a procedure under anesthesia. With medical practice becoming more and more tailored to the individual needs of the patient, we should be aware of this option for minimizing risks to the patient.

Bleeding and large or multiple findings were a leading cause of failed or incomplete procedures in the office, while in the OR they limited the procedure in only two cases. Bleeding in the uterine cavity limits the operating viewing field during hysteroscopy, but this problem is overcome in the OR by washing the uterine cavity continually with an irrigation flow pump using higher pressures than those used in the office. This also serves to explain the significant difference in the indications for which women were referred to the two different treatment options. More women were referred to office procedures due to abnormal sonographic findings, while more women with active PMB were referred to procedures in the OR. 
Our results revealed that cervical stenosis is not a contraindication for office hysteroscopy. We managed to overcome cervical stenosis in more than half of the patients who presented with it (32/54 cases, 59\%). The techniques used in our clinic to deal with cervical stenosis are either mechanical, including forcing pressure with the hysteroscope or using scissors, or electrical, using a bipolar electrode to widen the orifice and penetrate through the fibrotic tissue. Pretreatment with $25 \mathrm{mcg}$ of vaginal estradiol in combination with $400 \mathrm{mcg}$ of vaginal misoprostol before the procedure can facilitate passage of the hysteroscope through the cervical canal [13].

The most compelling advantage of office hysteroscopy compared to OR hysteroscopy in this cohort is the absence of complications in the former and the higher rate and severity of complications in the latter, including ICU hospitalization, sepsis, abdominal operation, and two mortalities related to the OR hysteroscopy procedure. A similar recent publication on office hysteroscopy in postmenopausal women did not observe any complications, such as uterine infection, uterine perforation, or genital tract hemorrhage, either [11].

The volume of procedures in the office increased 16 times during the study period, and a higher percentage of malignancies was diagnosed in the office procedures over time (Fig. 1B). These trends are the result of a newly implemented technique in our institution, and also of a change of clinical practice of referring women to the office option as a first-line procedure. Once the refined biopsy-taking technique was implemented, no significant change in yield was observed during the last three years of the study, indicating that the technique is relatively quickly and easily learned and put into practice.

Malignancy was found in $30.9 \%$ of the patients who presented with PMB, as well as in $7.3 \%$ of the patients who had no PMB. The pathological results from the repeat hysteroscopy are striking. One-quarter $(12 / 48)$ of the samples retrieved from repeat hysteroscopy were abnormal. This emphasizes the importance of pursuing a diagnosis. In selected cases of a moderate-to-high index of suspicion, we do not favor a conservative sonographic follow-up but rather recommend referring the patient directly to a repeat hysteroscopy. It is noteworthy that malignancy was detected in $6 / 36(16.7 \%)$ of the patients who did not present with PMB and who were referred to a repeat hysteroscopy.

Our study has several limitations. The main limitation is that the two sub-groups were not matched for demographic or clinical characteristics or for indications for undergoing the procedure. Also, we did not have information about previous procedures that may have been performed outside our medical center. It is likely that some patients with a history of failure of a previous trial of office procedures were directly referred to OR hysteroscopy. While our data are primarily descriptive, they nevertheless suggest that office hysteroscopy for women over the age of 70 is safe, feasible, and reliable. Moreover, to the best of our knowledge, this is the first study to compare office and OR hysteroscopy for this age group. We believe that patient selection will improve with greater implementation of office hysteroscopy and as more and more physicians become aware of the technique's advantages as well as its limitations. This, in turn, will lead to higher rates of successfully completed procedures and to higher rates of accurate pathological 
diagnoses in the office setting. Further prospective trials are needed in order to confirm our findings. Such studies may help to design a tool for better patient selection for either office or OR hysteroscopy.

\section{Conclusion}

The technologies currently available for office-based hysteroscopy are advantageous for patients 70 years of age or older in order to avoid the risks of anesthesia and to promote lower complication rates and fair chances of a complete procedure. In patients with failed hysteroscopy and a moderate-to-high index of suspicion of malignancy, a repeat hysteroscopy is recommended. Trends over time have enabled the achievement of significant numbers of abnormal findings in the office, making the office procedure a viable option, especially for a patient population characterized by higher rates of clinically significant findings.

\section{Abbreviations}

Operating room (OR)

Postmenopausal bleeding (PMB)

Interquartile range (IQR)

Intensive care unit (ICU)

Intrauterine device (IUD)

\section{Declarations}

Ethics approval and consent to participate: This study was approved by the Research Ethics Committee at the Tel Aviv Sourasky Medical Center. The need for consent to participate was waived by an IRB (074915-TLV on 10.11.2016).

Consent for publication: not applicable

Availability of data and material: The database used and / or analysed during the current study are available from the corresponding author on reasonable request.

Competing interests: The authors declare that they have no competing interests.

Funding: The authors declare that they have not received funding for this research.

Authors contributions: S.B. and G.R. performed the office hysteroscopies and, together with N.S., collected data . S.B., N.S and D.G. wrote the text of the manuscript, analyzed the data and prepared the figures. A.B.D analyzed the data and prepared the figures. All authors reviewed and approved the final manuscript. 
Acknowledgements: not applicable.

\section{References}

1. ACOG Committee Opinion No. 426: The Role of Transvaginal Ultrasonography in the Evaluation of Postmenopausal Bleeding. Obstet Gynecol. 2009;113:462-4. doi:10.1097/AOG.0b013e31819930cc.

2. Giannella L, Mfuta K, Setti T, Boselli F, Bergamini E, Cerami LB. Diagnostic accuracy of endometrial thickness for the detection of intra-uterine pathologies and appropriateness of performed hysteroscopies among asymptomatic postmenopausal women. Eur J Obstet Gynecol Reprod Biol. 2014;177:29-33.

3. Di Spiezio Sardo A, Taylor A, Tsirkas P, Mastrogamvrakis G, Sharma M, Magos A. Hysteroscopy: a technique for all? Analysis of 5,000 outpatient hysteroscopies. Fertil Steril. 2008;89:438-43.

4. Serden SP. Diagnostic hysteroscopy to evaluate the cause of abnormal uterine bleeding. Obstet Gynecol Clin North Am. 2000;27:277-86.

5. Gebauer G, Hafner A, Siebzehnrübl E, Lang N. Role of hysteroscopy in detection and extraction of endometrial polyps: Results of a prospective study. Am J Obstet Gynecol. 2001;184:59-63. doi:10.1067/mob.2001.108332.

6. Campo R, Van Belle Y, Rombauts L, Brosens I, Gordts S. Office mini-hysteroscopy. 1999.

7. Nagele F, Bournas N, O'Connor H, Broadbent M, Richardson R, Magos A. Comparison of carbon dioxide and normal saline for uterine distension in outpatient hysteroscopy. Fertil Steril. 1996;65:305-9.

8. Bettocchi S, Selvaggi L. A vaginoscopic approach to reduce the pain of office hysteroscopy. 1997.

9. Bar-On S, Ben-David A, Rattan G, Grisaru D. Is outpatient hysteroscopy accurate for the diagnosis of endometrial pathology among perimenopausal and postmenopausal women? Menopause. 2018;25:160-4.

10. Giampaolino P, Corte L Della, Filippo C Di, Mercorio A, Vitale SG, Bifulco G, et al. Office hysteroscopy in the management of women with postmenopausal bleeding. Climacteric. 2020;23:369-75. doi:10.1080/13697137.2020.1754389.

11. Sauvan M, Pourcelot A-G, Fournet S, Fernandez H, Capmas P. Office hysteroscopy for postmenopausal women: Feasibility and correlation with transvaginal ultrasound. J Gynecol Obstet Hum Reprod. 2018;47:505-10. doi:10.1016/j.jogoh.2018.08.018.

12. Sousa R, Silvestre M, Almeida L, Sousa E, Falca o F, Falca o F, et al. Acta Obstetricia et Gynecologica Scandinavica Transvaginal ultrasonography and hysteroscopy in postmenopausal bleeding a prospective study. Acta Obs Gynecol Scand. 2001;80:856-62.

13. Oppegaard KS, Lieng M, Berg A, Istre O, Qvigstad E, Nesheim BI. A combination of misoprostol and estradiol for preoperative cervical ripening in postmenopausal women: A randomised controlled trial. BJOG An Int J Obstet Gynaecol. 2010;117:53-61. 


\section{Figures}

A

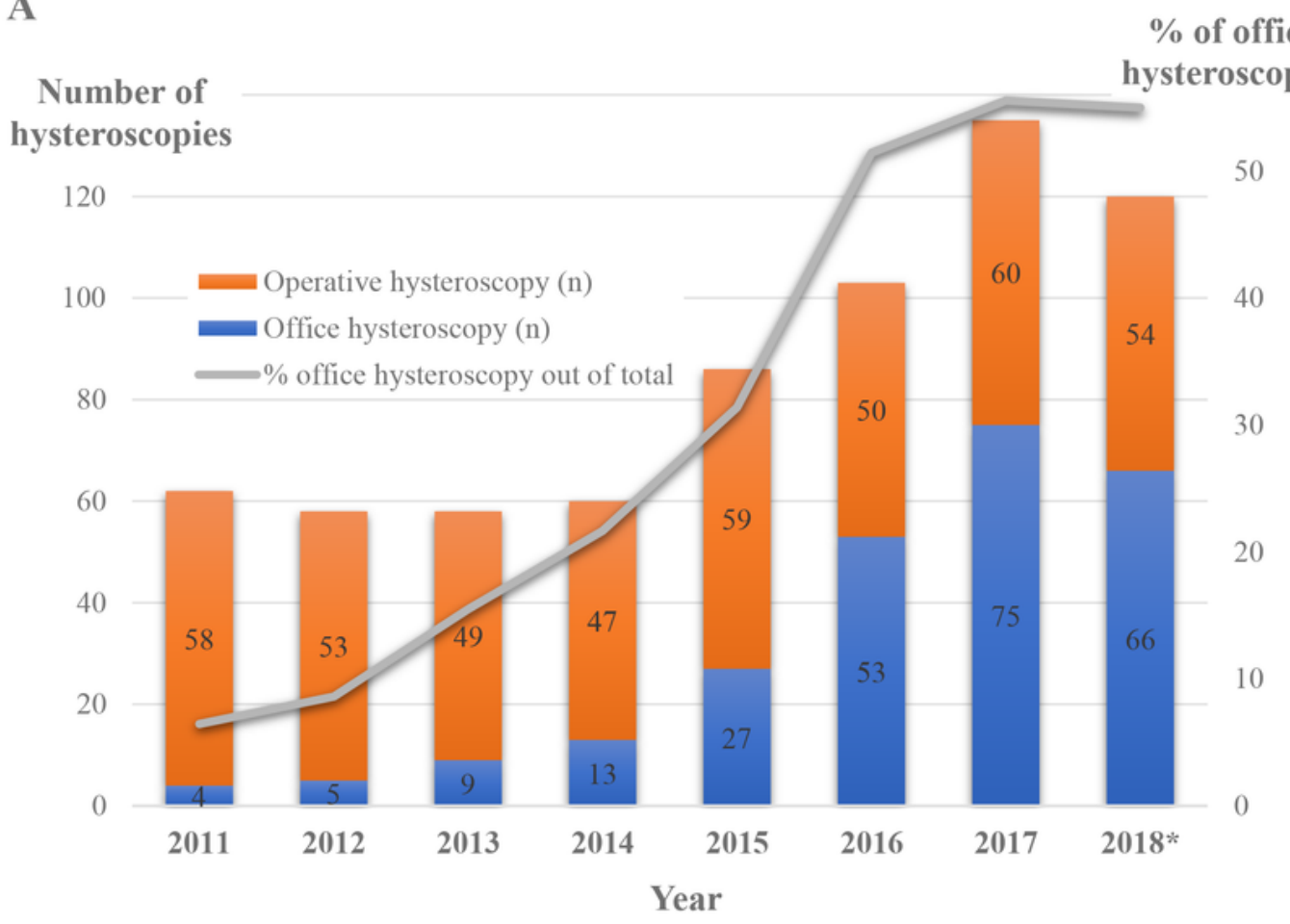

B

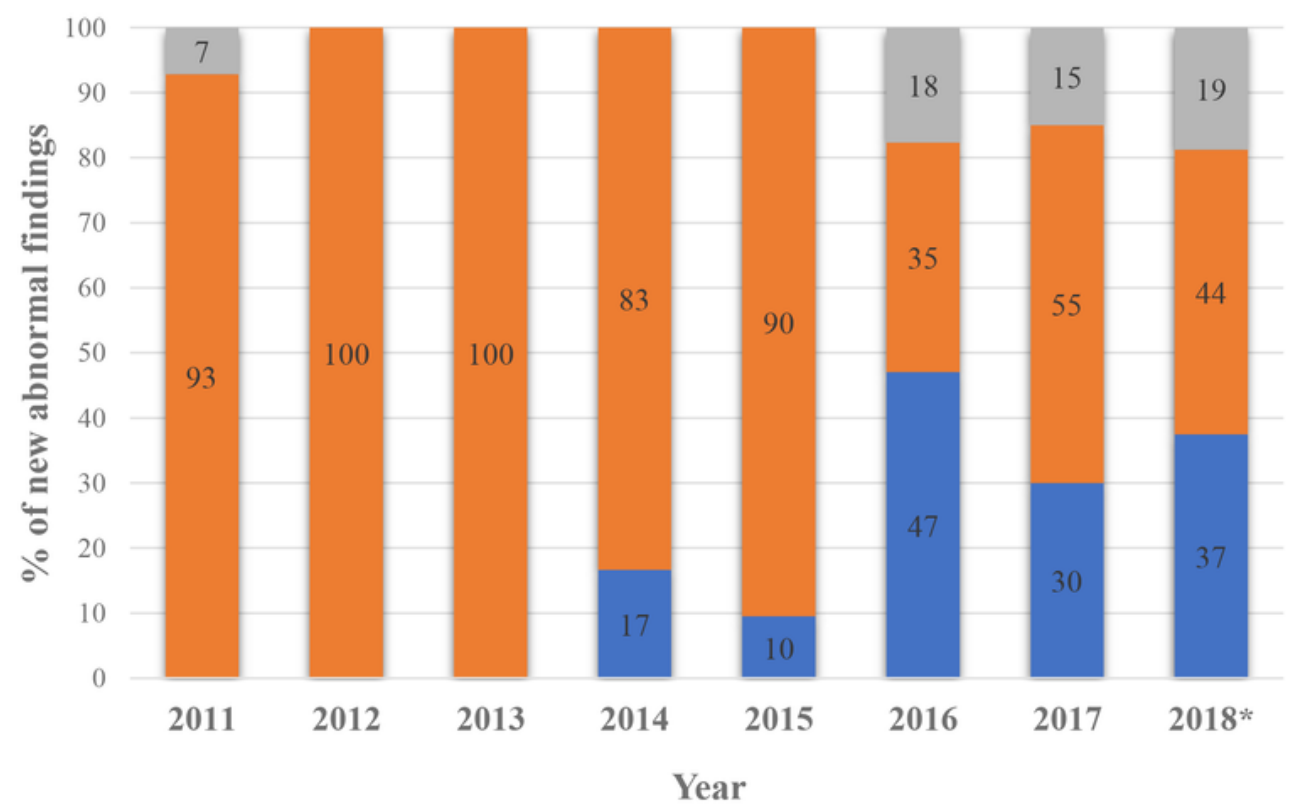

- Office hysteroscopy $\quad$ Operating room hysteroscopy $\quad$ Second hysteroscopy

\section{Figure 1}

Trends in the utility of hysteroscopy techniques and the occurrence of abnormal findings for women 70 years and older during the study period $A$. The annual volume of activity according to the type of procedure $\mathrm{B}$. The annual diagnosis of abnormal findings according to the type of procedure (\%) *Based 
on data collected until August 31, 2018. Second hysteroscopy- hysteroscopy performed after failed or incomplete office hysteroscopy 